\title{
Left ventricular pumping during the transition-adaptation sequence in preterm infants: impact of the patent ductus arteriosus
}

Sigrid Baumgartner ${ }^{1}$, Monika Olischar ${ }^{1}$, Martin Wald ${ }^{2}$, Tobias Werther ${ }^{1}$, Angelika Berger $^{1}$, Thomas Waldhör $^{3}$, Georg Fischer ${ }^{4}$ and Ulrike Salzer-Muhar ${ }^{5}$

BACKGROUND: Postnatally, the immature left ventricle (LV) is subjected to high systemic afterload. Hypothesizing that LV pumping would change during transition-adaptation, we analyzed the LV in preterm infants $(G A \leq 32+6)$, clinically stable or with a hemodynamically significant patent ductus arteriosus (hPDA) by applying a pump model.

METHODS: Pumping was characterized by $E_{\mathrm{A}}$ (effective arterial elastance, reflecting afterload), $E_{\mathrm{ES}}$ (end-systolic LV elastance, reflecting contractility), $E_{A} / E_{E S}$ coupling ratios, descriptive $E_{\mathrm{A}}: E_{\mathrm{ES}}$ relations, and $E_{\mathrm{A}} / E_{\mathrm{ES}}$ graphs. Data calculated from echocardiography and blood pressure were analyzed by diagnosis (S group: clinically stable, no hPDA, $n=122$; hPDA group, $n=53$ ) and by periods (early transition: days of life 1-3; late transition: 4-7; and adaptation: 8-30).

RESULTS: $S$ group: LV pumping was characterized by an increased $E_{A} / E_{E S}$ coupling ratio of 0.65 secondary to low $E_{E S}$ in early transition, a tandem rise of both $E_{\mathrm{A}}$ and $E_{\mathrm{ES}}$ in late transition, and an $E_{A} / E_{E S}$ coupling ratio of 0.45 secondary to high $E_{E S}$ in adaptation; hPDA group: time-trend analyses showed significantly lower $E_{\mathrm{A}}(P<0.0001)$ and $E_{\mathrm{ES}}(P=0.006)$. Therefore, LV pumping was characterized by a lower $E_{A} / E_{E S}$ coupling ratio $(P=0.088)$ throughout transition-adaptation.

CONCLUSIONS: In stable infants, facing high afterload, the immature LV, enhanced by the physiological PDA, increases its contractility. In hPDA, facing low afterload, the overloaded immature LV exhibits a consistently lower contractility.

$\mathrm{n}$ term infants, immediate cardiovascular transition at birth is followed by an early-transition phase (change from a right-to-left to a left-to-right shunt (LRS) across the patent ductus arteriosus (PDA)), a late-transition phase (oxygenmediated PDA constriction by days of life 2-3), and eventually, an adaptation phase (at the end of the first and subsequent weeks of life (left ventricular and vascular remodeling) (1).
In preterm infants, this physiological cardiovascular transition-adaptation sequence has to proceed with altered responses in the systemic and pulmonary vasculature $(2,3)$. While individual infants will exhibit near-to-physiologic hemodynamics within a slightly prolonged sequence, others will exhibit pathophysiologic hemodynamics, such as a hemodynamically significant PDA (hPDA) with cardiac and pulmonary volume overload $(4,5)$, as well as altered cerebral perfusion (6).

The left ventricle (LV) of the preterm infant has to pump an appropriate stroke volume (SV) while being prematurely exposed to a high systemic afterload. According to published knowledge, the immature LV exhibits an inherently low contractility and a particular sensitivity to increases in afterload (7).

\section{The Immature LV}

Before birth, the fetal LV pumps into the aorta up to the watershed of the isthmus, its two respective sources of filling being the foramen ovale and the pulmonary blood flow. In the sarcomere, the fetal isoform of titin is less stiff than the postnatal form (7). The giant myofilament protein titin is the largest protein known and spans a continuous filament network across the sarcomere. Titin, by acting as a molecular spring, defines the passive elasticity of the cardiomyocyte in diastole (8). The compliant fetal titin isoform enables the heart to grow and to generate an adequate output even at the very low filling pressures in utero $(7,9)$.

In late gestation, an inverse correlation between foramen ovale shunt flow and pulmonary blood flow has been noted (10). It is very likely that the augmentation of pulmonary blood flow (reaching up to $30 \%$ of the combined ventricular output) is driven by variations in pulmonary vascular resistance (10).

Concomitantly, both the increasing LV Doppler early diastolic filling rates (11) and decreasing isovolumetric

\footnotetext{
${ }^{1}$ Department of Pediatrics and Adolescent Medicine, Division of Neonatology, Pediatric Intensive Care and Neuropediatrics, Medical University of Vienna, Vienna, Austria; ${ }^{2}$ Department of Pediatrics and Adolescent Medicine, Division of Neonatology, Paracelsus Medical University, Salzburg, Austria; ${ }^{3}$ Department of Epidemiology, Medical University of Vienna, Vienna, Austria; ${ }^{4}$ Section for Medical Information Management and Imaging, Medical University of Vienna, Austria; ${ }^{5}$ Department of Pediatrics and Adolescent Medicine, Division of Pediatric Cardiology, Medical University of Vienna, Vienna, Austria. Correspondence: U Salzer-Muhar (ulrike.salzer@meduniwien.ac.at) Received 11 September 2017; accepted 5 February 2018; advance online publication 11 April 2018. doi:10.1038/pr.2018.22
} 


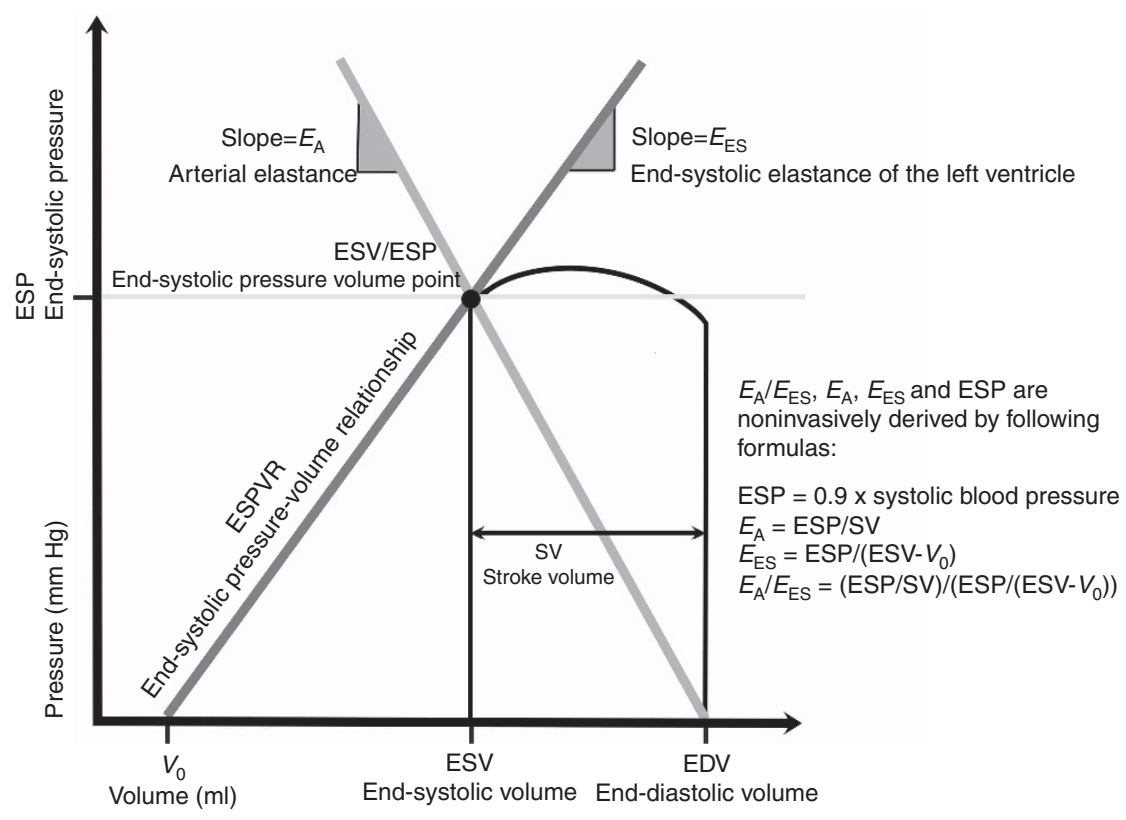

Figure 1. Pressure-volume relationship. Arterial elastance $\left(E_{\mathrm{A}}\right)$ is a measure of left ventricle (LV) afterload and is the slope of the line passing the $x$ axis intercept end-diastolic volume (EDV) and the end-systolic volume (ESV)/end-systolic pressure (ESP) point. ESP volume relation (ESPVR) provides a load-independent measure of contractile function and is defined by the slope $E_{\mathrm{ES}}$ and $V_{0}$. $E_{\mathrm{ES}}$ quantifies $\mathrm{LV}$ elastance at the end systole.

relaxation time (12) indicate an improvement in relaxation and subsequently an increased LV preload.

An increased preload enhances systolic performance and thus SV. This is known as the Frank-Starling mechanism. In the sarcomere, it is again the giant myofilament protein titin that governs this mechanism (8). By regulating the length of the thick myosin filament, titin defines muscular force and how this force varies with muscle length (13).

The stiff postnatal titin isoform promotes, and the compliant fetal titin isoform attenuates the Frank-Starling mechanism (8). With term birth, the LV will be already prepared to pump an appropriate SV. With preterm birth, however, the lack of late gestation filling and the presence of the fetal titin isoform could attenuate the systolic function of the immature, inadequately preloaded LV.

As we hypothesized that the systolic pump function of the immature LV would change during transition-adaptation, we set up this study to explore the LV throughout this unique period. To include the effect of physiological and pathophysiological ductal patency on afterload, we searched for a method that would enable us to assess the LV, and concomitantly, its systemic afterload in preterm infants.

\section{Ventricular-Arterial Coupling}

The theoretical pump model ventricular-arterial coupling $\left(E_{\mathrm{A}} / E_{\mathrm{ES}}\right)$ of Sunagawa et al. (14) allows analysis of the systolic pump function of the LV in its context to the arterial system (15). While $E_{\mathrm{A}}$, effective arterial elastance, characterizes the net afterload, $E_{\mathrm{ES}}$, end-systolic chamber elastance, is a loadindependent descriptor of LV contractility that is also influenced by LV geometry, end-systolic stiffness, and myocardial properties $(16,17)$.
The gold standard for estimating the components $E_{\mathrm{A}}$ and $E_{\mathrm{ES}}$ is the invasively obtained pressure-volume relationship (Figure 1, a modified version of Chantler et al. (18)). $E_{\mathrm{A}}$ can be derived by the end-systolic pressure (ESP)-stroke-volume relationship and $E_{\mathrm{ES}}$ by the ESP-volume relationship. Invasive evaluation is not feasible in the fragile patient group of preterm infants. However, the sophisticated and diverse methodology (18-21) to estimate LV pump function from echocardiographic parameters (end-systolic volume (ESV), $\mathrm{SV}$, and from blood pressure (BP)) that has already been applied in both adults $(17,22)$ and children $(19,20,23,24)$ appeared to be a noninvasive alternative for preterm infants.

We decided to use the $E_{\mathrm{A}} / E_{\mathrm{ES}}$ model to explore the systolic pump function of the immature ventricle during transitionadaptation. The aim of this study was to assess LV pumping during the transition-adaption sequence in stable preterm infants with near-to-physiologic hemodynamics and also in sick infants exhibiting the pathophysiologic hemodynamics of a hPDA.

\section{METHODS}

We used data collected for a prospective study on PDA treatment. Examinations were performed following our functional Echocardiography (fEcho) Protocol established in 2009, which is in line with published recommendations (25). Our setting of fEcho also includes prospectively standardized electronic fEcho Report Forms that ensure automatic insertion of the infants' demographic data, heart rate, $\mathrm{BP}$, and oxygen saturation at the moment of examination. Drop downs allow linking of measurements immediately to pretermrelevant hemodynamics. Report forms, images, and loops are archived in our fEcho Digital Platform (created by the Section for Medical Information Management and Imaging by the Medical University of Vienna, Austria). 


\section{Articles | Baumgartner et al.}

\section{Study Population}

The inclusion criteria were very preterm infants born between $23+0$ and $32+6$ weeks of gestation, diagnosis of near-to-physiologic hemodynamics or hPDA, and normal intracardiac anatomy.

We formed groups by diagnosis: (i) hPDA group: a pure LRS across the PDA and an end-diastolic flow velocity in the left pulmonary artery (LPAd) $\geq 0.2 \mathrm{~m} / \mathrm{s}$ (4), which—with normal-sized pulmonary arteries-indicates a significant LRS into the pulmonary vasculature. (ii) S group: clinically stable, with either a closed PDA or a non-hPDA (LPAd $<0.2 \mathrm{~m} / \mathrm{s}$, ratio of the left atrial diameter to the aortic diameter $<1.4$, and no abdominal aortic backward flow (5)).

To represent the phases of the transition-adaptation sequence, we split the results within each group by periods based on the days of life (DOL) on which the examinations were performed: period 1: DOL 1-3 (early transition), period 2: DOL 4-7 (late transition), and period 3: DOL 8-30 (adaptation). By defining those time frames, which slightly exceeded the established term infants' time frames, we aimed to take into account the assumed prolongation of the transitionadaptation sequence in preterm infants.

If infants had more than one examination in one period, we only included the first examination.

\section{Data}

Weight, heart rate, transcutaneous oxygen saturation, and BP (measured invasively if a catheter was in place) were retrieved from electronic fEcho Report Forms. BP was measured invasively if a catheter was in place or noninvasively.

End-diastolic volume (EDV), ESV, and SV were retrieved from the fEcho Digital Platform. These volumes, based on M-mode measurements of the end-diastolic and end-systolic diameters of the LV (parasternal long axis view) are provided by the software of the ultrasound machine (Siemens, Acuson S2000) using the Teichholz formula (26).

\section{Systolic Pump Function}

Regarding $E_{\mathrm{A}}$ and $E_{\mathrm{ES}}$ and the so-called $E_{\mathrm{A}} / E_{\mathrm{ES}}$ coupling ratio, we refer to Figure 1 (18). To apply the pump model and its formulas which were developed for adults (14) in preterm infants, we estimated $E_{\mathrm{A}}, E_{\mathrm{ES}}$, and ESP by the following formulas: $\mathrm{ESP}=0.9 \times$ systolic $\mathrm{BP}(20,27,28) ; E_{\mathrm{A}}=\mathrm{ESP} / \mathrm{SV}(14)$; and $E_{\mathrm{ES}}=\mathrm{ESP} /\left(\mathrm{ESV}-V_{0}\right)$. $V_{0}$ is the left ventricular volume at a theoretical (nonphysiological) ESP in the LV of $0 \mathrm{~mm} \mathrm{Hg}(29,30)$. If we assume that $V_{0}$ is small compared with ESV (18), then $V_{0}$ might be neglected, and the calculation of $E_{\mathrm{ES}}$ reduces to $E_{\mathrm{ES}}=\mathrm{ESP} / \mathrm{ESV}(18-20)$.

The following approach-related limitations have to be considered:

(i) BP data included not only invasive measurements but also noninvasive measurements. The degree of agreement between the two methods may vary, but there may be substantial differences in individual preterm infants (31). This is also depicted by our own preliminary data obtained from 369 preterm infants with 918 examinations (Supplementary Figure S1 (online), T. Werther et al.).

(ii) Calculation of ESP by $0.9 \times$ systolic BP may slightly underestimate the ESP subject to the contour of the individual LV pressure curve in the hPDA group.

(iii) The Teichholz method overestimates both EDV and ESV, and thus leads to a significantly higher SV compared with SVs calculated by other methods $(28,32)$. Aiming to compare $E_{\mathrm{A}}, E_{\mathrm{ES}}$, and $E_{\mathrm{A}} / E_{\mathrm{ES}}$ between groups, we considered this systematic bias as being acceptable. In premature infants, obtaining a true apical fourchamber view for LV volumetric chamber quantification is not always feasible.

(iv) In a recent validation study performed in children with nearto-normal loading conditions, 3D echocardiographic estimated $E_{\mathrm{ES}}$ correlated best with invasively measured $E_{\mathrm{ES}}$ when $V_{0}$ was neglected (20). This was attributed to the high interobserver variability in the measurement of the pre-ejection period (required for the calculation of $V_{0}$ ) in the presence of high heart rates. In hPDA pathophysiology, however, when $V_{0}$ is increased, neglecting of $V_{0}$ may lead to underestimation of $E_{\mathrm{ES}}$ and consecutively, to overestimation of $E_{\mathrm{A}} /$ $E_{\mathrm{ES}}$.

(v) The $E_{\mathrm{A}} / E_{\mathrm{ES}}$ graphs (Figure 2) were drawn by using the equation of a straight line $\left(\mathrm{ESP}=E_{\mathrm{ES}} \times \mathrm{ESV}+d_{\mathrm{Ees}}\right.$ and $\left.\mathrm{ESP}=E_{\mathrm{A}} \times \mathrm{ESV}+d_{\mathrm{Ea}}\right)$. The mean values of $E_{\mathrm{ES}}, E_{\mathrm{A}}$, and the ESP volume point (ESP/ESV) were used to calculate $d\left(d_{\mathrm{Ees}}\right.$ and $\left.d_{\mathrm{Ea}}\right)$ and to draw the straight lines. Thus, the $E_{\mathrm{A}} / E_{\mathrm{ES}}$ graphs represent the mean values for $E_{\mathrm{A}}, E_{\mathrm{ES}}$, and $\mathrm{ESP} / \mathrm{ESV}$, whereas $V_{0}$ and EDV result from the respective $x$ axis intercepts of the two lines.

\section{Statistics}

Mean values and standard deviation were used to describe infants' characteristics including clinical and echocardiographic parameters, as well as $E_{\mathrm{A}}, E_{\mathrm{ES}}$, and $E_{\mathrm{A}} / E_{\mathrm{ES}}$.

The effects of the variables "diagnosis" and "period" on $E_{\mathrm{A}}, E_{\mathrm{ES}}$, and $E_{\mathrm{A}} / E_{\mathrm{ES}}$ were estimated by means of a linear mixed model in SAS (SAS VS. 9.4 Institute Inc., Cary, NC, 2012). The variables "diagnosis" and "period" were treated as class effects. The a priori null hypothesis of the equality of time trends between the two diagnosis groups was tested using an interaction term diagnosis $\times$ period. $E_{\mathrm{A}}$, $\mathrm{E}_{\mathrm{ES}}$, and $E_{\mathrm{A}} / E_{\mathrm{ES}}$ were transformed using the natural logarithm to approach a normal residual distribution. The significance level was set to $0.05 / 3$ by means of the Bonferroni correction to adjust for multiple testing by estimating three regression models.

\section{Ethics}

This study (EC-no. 1079/2011) and also the prospective study on PDA treatment (EC-no. 875/2010) were approved by the Ethics Board of the Medical University of Vienna.
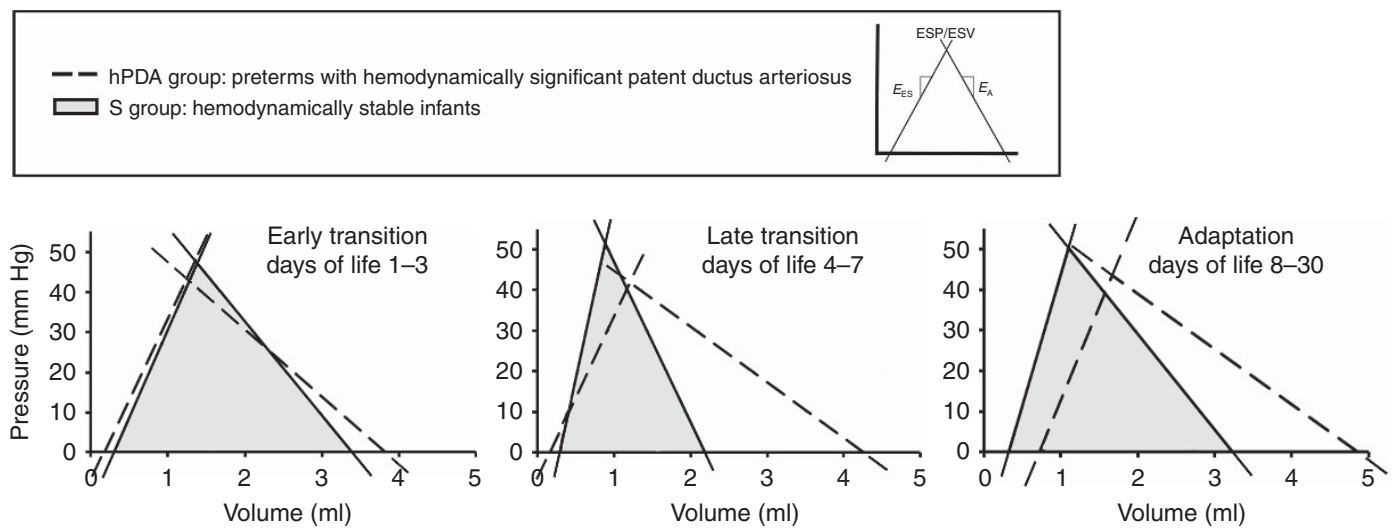

Figure 2. Comparison of the corresponding ventricular-arterial coupling $\left(E_{\mathrm{A}} / E_{\mathrm{ES}}\right)$ graphs. 


\section{RESULTS}

The study population included 126 preterm infants with a total number of 175 echocardiographic examinations, of which 122 were assigned to the $\mathrm{S}$ group and 53 to the
hPDA group. Actually, 40 preterm infants had multiple observations, 4 preterm infants crossed from the hPDA group to the $S$ group, and 12 patients in the hPDA group had serial measurements.

Table 1. Clinical and echocardiographic parameters of the $S$ and hPDA group

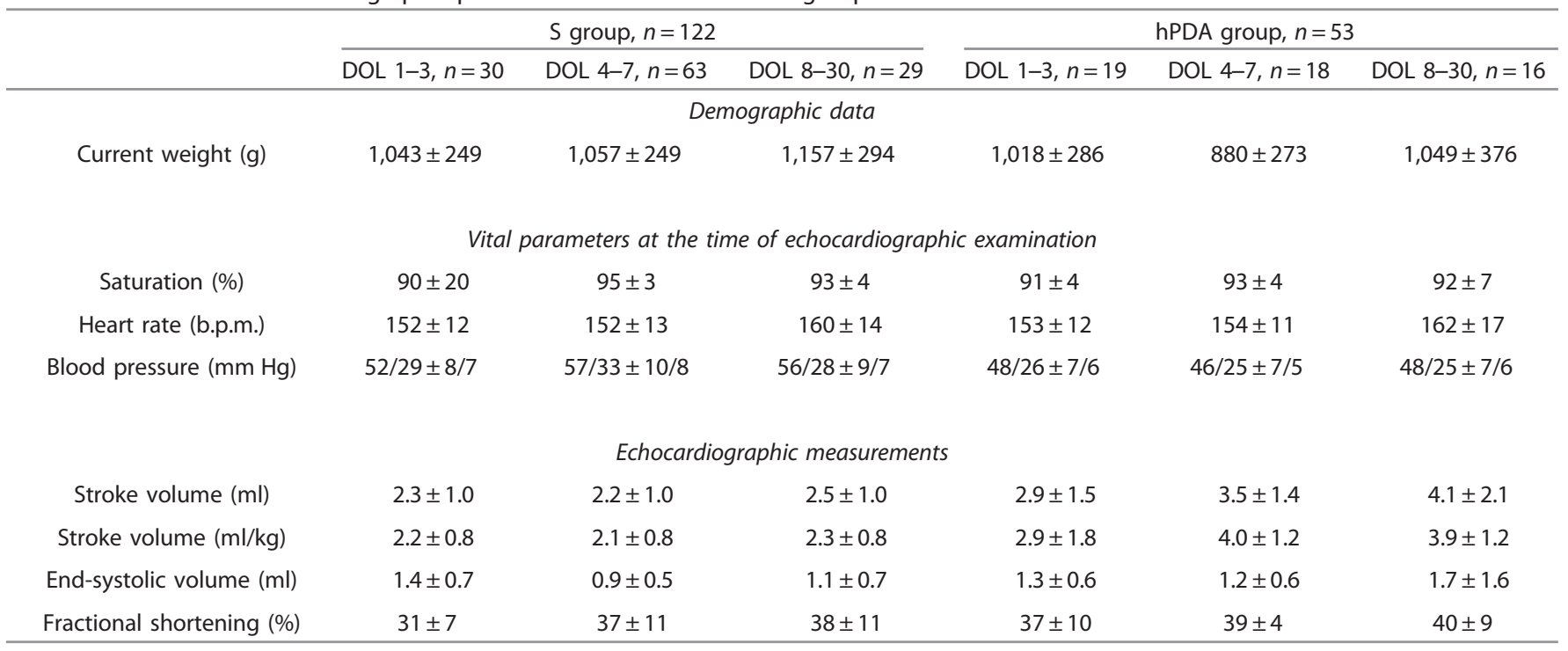

DOL, days of life; hPDA, hemodynamically significant patent ductus arteriosus; S, stable preterms.

Table 2. Comparison of $E_{\mathrm{A}} / E_{\mathrm{ES}}, E_{\mathrm{A}}$, and $E_{\mathrm{ES}}$ between the $S$ and $\mathrm{hPDA}$ group

\begin{tabular}{|c|c|c|c|c|c|c|c|c|}
\hline & \multicolumn{3}{|c|}{ S group, $n=122$} & \multicolumn{3}{|c|}{ hPDA group, $n=53$} & \multicolumn{2}{|c|}{$\begin{array}{l}P \text { values type III } \\
\text { tests of fixed } \\
\text { effects }\end{array}$} \\
\hline & DOL $1-3 n=30$ & DOL 4-7 $n=63$ & DOL $8-30 n=29$ & DOL $1-3 n=19$ & DOL 4-7 $n=18$ & DOL 8-30 $n=16$ & Diagnosis & Period \\
\hline$E_{\mathrm{A}} / E_{\mathrm{ES}}$ & $0.65 \pm 0.42$ & $0.56 \pm 0.61$ & $0.45 \pm 0.29$ & $0.49 \pm 0.35$ & $0.35 \pm 0.09$ & $0.39 \pm 0.21$ & 0.0876 & 0.0582 \\
\hline$E_{\mathrm{A}}(\mathrm{mm} \mathrm{Hg} / \mathrm{ml})$ & $24 \pm 10$ & $40 \pm 50$ & $24 \pm 10$ & $17 \pm 8$ & $14 \pm 6$ & $14 \pm 7$ & $<.0001$ & 0.2517 \\
\hline$E_{\mathrm{ES}}(\mathrm{mm} \mathrm{Hg} / \mathrm{ml})$ & $44 \pm 26$ & $85 \pm 81$ & $65 \pm 39$ & $40 \pm 15$ & $40 \pm 18$ & $46 \pm 33$ & 0.0062 & 0.0425 \\
\hline
\end{tabular}

$\mathrm{DOL}$, days of life; $E_{\mathrm{A}}$, arterial elastance; $E_{\mathrm{A}} / E_{\mathrm{ES}}$, ventricular-arterial coupling; $E_{\mathrm{ES}}$, end systolic elastance of the left ventricle; $\mathrm{hPDA}$, hemodynamically significant patent ductus arteriosus; $S$, stable preterms.

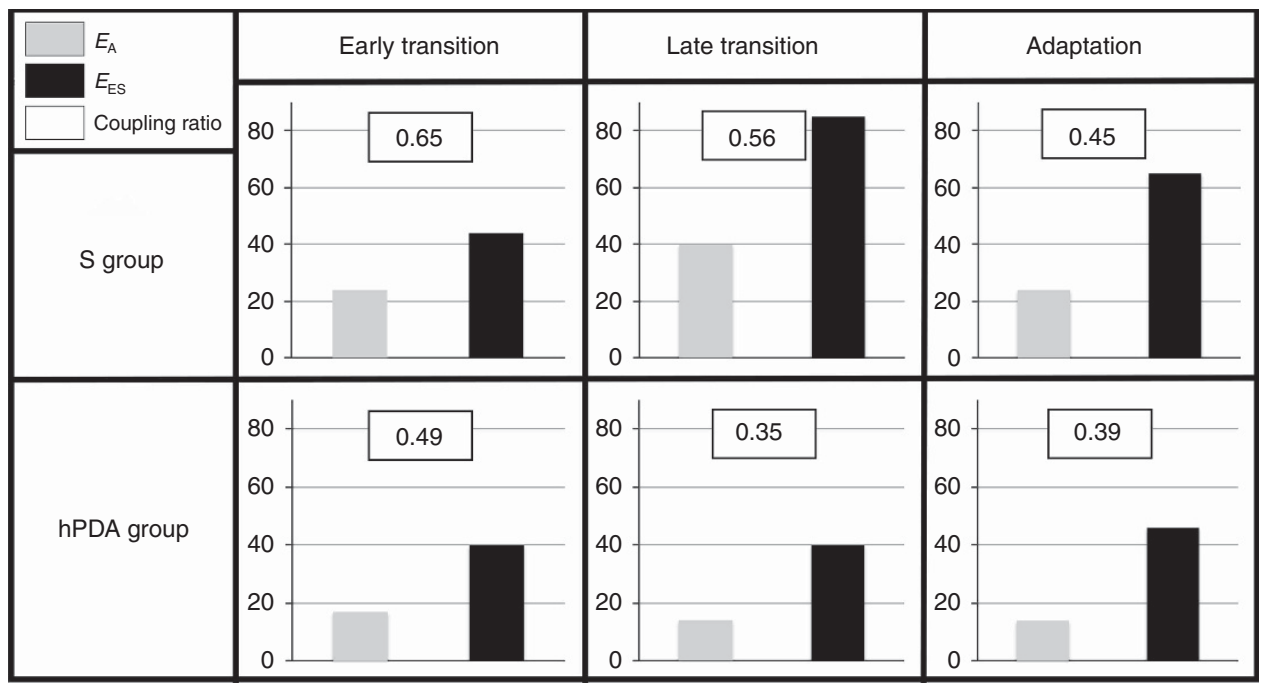

Figure 3. Ventricular-arterial coupling $\left(E_{\mathrm{A}}: E_{\mathrm{ES}}\right)$ relations and their changes during transition-adaptation. 


\section{Articles | Baumgartner et al.}

A non-hPDA was present in the S-group at $67 \%, 46 \%$, and $10 \%$ in the periods 1,2 , and 3 , respectively.

\section{Comparison of the S Group vs. the hPDA Group}

Table 1 presents descriptive statistics of clinical and echocardiographic parameters in the two groups. Oxygen saturation (except in period 1), and systolic and diastolic BP were lower in the hPDA group.

Table 2 shows results of the comparison of $E_{\mathrm{A}} / E_{\mathrm{ES}}, E_{\mathrm{A}}$, and $E_{\mathrm{ES}}$. The interaction term diagnosis $\times$ period, being not significant, indicated a lack of effect of period between the two groups. $E_{\mathrm{ES}}(P=0.0062)$ and $E_{\mathrm{A}}(P<0.0001)$ were significantly lower in the hPDA group, whereas $E_{\mathrm{A}} / E_{\mathrm{ES}}$ was not.

Descriptive Analysis. S group: $E_{\mathrm{A}}$ was comparable in periods 1 and 3. $E_{\mathrm{ES}}$ was at minimum in period $1 . E_{\mathrm{A}} / E_{\mathrm{ES}}$ coupling ratios show a gradual decrease with 0.65 in period $1,0.56$ in period 2, and 0.45 in period 3 (Figure 3).

$h P D A$ group: $E_{\mathrm{A}}$ was consistently low in periods 1,2 , and 3 . $E_{\mathrm{ES}}$, comparable to the minimum of $E_{\mathrm{ES}}$ in the $\mathrm{S}$ group, was low in periods 1 and 2 , and at its maximum in period $3 . E_{\mathrm{A}} /$ $E_{\mathrm{ES}}$ coupling ratios were 0.49 in period $1,0.35$ in period 2, and 0.39 in period 3 , respectively.

$\boldsymbol{E}_{\mathrm{A}} / \boldsymbol{E}_{\mathrm{ES}}$ Graphs. The corresponding $E_{\mathrm{A}} / E_{\mathrm{ES}}$ graphs (Figure 2) for the $S$ group showed higher ESP levels (due to higher systolic $\mathrm{BP}$ ), whereas the corresponding $E_{\mathrm{A}} / E_{\mathrm{ES}}$ graphs for the hPDA group showed an expansion and a rightward shift due to low $E_{\mathrm{A}}$ and $E_{\mathrm{ES}}$ (resulting from high SV and ESV values).

\section{DISCUSSION}

\section{Advantages of the $\mathrm{E}_{\mathrm{A}} / \mathrm{E}_{\mathrm{ES}}$ Pump Model}

There are two reasons why we chose the $E_{\mathrm{A}} / E_{\mathrm{ES}}$ pump model to analyze LV systolic pump function within the transitionadaptation sequence:

First, the $E_{\mathrm{A}} / E_{\mathrm{ES}}$ coupling ratio is inversely related to the ejection fraction $\left(E_{\mathrm{A}} / E_{\mathrm{ES}}=1 /\right.$ (ejection fraction-1)) with the additional advantage that by examining $E_{\mathrm{A}}$ and $E_{\mathrm{ES}}$ separately, alterations can be attributed to alterations in arterial or ventricular function, or both (18). Generally, $E_{\mathrm{ES}}$ is matched with $E_{\mathrm{A}}$, and more specifically, $E_{\mathrm{ES}}$ responds to a given $E_{\mathrm{A}}$. Various conditions, however, may lead to an acute mismatch between the arterial and ventricular systems (18). During exercise for example, a disproportionate increase occurs in $E_{\mathrm{ES}}$ vs. $E_{\mathrm{A}}$ due to an increase in left ventricular output caused by the higher speed and force of the left ventricular contractility (17). Corresponding to this acute mismatch, the $E_{\mathrm{A}} / E_{\mathrm{ES}}$ coupling ratio decreases.

Second, the $E_{\mathrm{A}} / E_{\mathrm{ES}}$ coupling ratio describes cardiac energetics. The efficiency of the ventricle as a pump is defined by the ratio between mechanical work (stroke work) and energy consumption (amount of oxygen the LV consumes while performing stroke work) (33). In adults, maximal energetic efficiency occurs when the $E_{\mathrm{A}} / E_{\mathrm{ES}}$ coupling ratio is close to 0.5 and maximal efficacy (stroke work) occurs when $E_{\mathrm{A}} / E_{\mathrm{ES}}$ coupling ratio is close to $1.0(10,13)$. In adults at rest, an optimal $E_{\mathrm{A}} / E_{\mathrm{ES}}$ coupling ratio between 0.7 and 1 seems to reflect the optimal balance between stroke work and energy consumption (17).

Before interpreting our results, we would like to point out that in children, due to smaller vessels and a higher heart rate, numeric values for $E_{\mathrm{A}}$ and $E_{\mathrm{ES}}$ are higher (17). They are inversely related to the body surface area (34). As $E_{\mathrm{ES}}$ values are by proportion higher in children than in adults, numeric $E_{\mathrm{A}} / E_{\mathrm{ES}}$ coupling ratios are lower. Published estimates for $E_{\mathrm{A}} /$ $E_{\mathrm{ES}}$ coupling ratios are 0.7 (34) and 0.6 (24) in children, and 0.5 in term infants, respectively (23).

\section{Estimated $\mathrm{E}_{\mathrm{A}} / \mathrm{E}_{\mathrm{ES}}$ Coupling Ratios in the Periods of the Transition-Adaptation Sequence}

In stable preterm infants, we observed a gradual decrease of estimated $E_{\mathrm{A}} / E_{\mathrm{ES}}$ from 0.65 in early transition over 0.56 in late transition to 0.45 in adaptation. We think that defining the three periods by the chosen time frames might have contributed to the nonsignificance of these results, as the length of these periods may vary individually.

The result, however, proved our hypothesis. It implies that in stable preterm infants with near-to-physiologic hemodynamics, the systolic pump function of the immature LV changes within the phases of the physiological transitionadaptation sequence. Besides, the value of 0.45 in adaptation, due to a proportionally even higher $E_{\mathrm{ES}}$ (Figure 3), corresponds well to the reported estimates in children.

With respect to the aforementioned cardiac energetics, the observed gradual decrease of the $E_{\mathrm{A}} / E_{\mathrm{ES}}$ coupling ratios indicates that in near-to-physiologic hemodynamics, the immature LV performs high stroke work in early transition and becomes more energy-efficient toward adaptation.

In preterm infants with hPDA, the consistently, albeit not significantly lower $E_{\mathrm{A}} / E_{\mathrm{ES}}$ coupling ratios throughout all periods may indicate a shift toward more LV energy efficiency (in response to the increased oxygen demand of the myocardium) at the expense of stroke work $(15,33)$. Our results are in line with the significantly lower $E_{\mathrm{A}} / E_{\mathrm{ES}}$ coupling ratios found (using a different methodology and definition of the PDA profile) in a recent study in preterm infants with an at least 14 days of exposure to a PDA (28).

\section{$\mathrm{E}_{\mathrm{A}}: \mathrm{E}_{\mathrm{ES}}$ Relations in the Three Periods of the Transition- Adaptation Sequence}

Examining $E_{\mathrm{A}}: E_{\mathrm{ES}}$ relations (Table 2 and Figure 3) and $E_{\mathrm{A}} /$ $E_{\mathrm{ES}}$ graphs (Figure 2) provided comprehensive insight into LV pumping in the respective days of life.

Stable preterm infants. In early transition, LV pumping was characterized by a maximum $E_{\mathrm{A}} / E_{\mathrm{ES}}$ coupling ratio secondary to a low $E_{\mathrm{ES}}$. A non-hPDA was present in $67 \%$ of these infants.

Our findings are in line with published data. They confirm that in this period, BP is kept up by the high systemic vascular resistance and not by SV (35), and also that the contractility of the immature myocardium is inherently low (7). 
In late transition, LV pumping was characterized by a tandem rise of $E_{\mathrm{A}}$ and $E_{\mathrm{ES}}$. The PDA was already closed in $54 \%$ of these infants. Higher $E_{\mathrm{A}}$, due to a higher systolic BP, indicated a higher afterload. $E_{\mathrm{ES}}$ was higher due to better contractility (36) and altered the LV geometry (Figure 2). The observed tandem rise indicates better LV contractility in response to the increased afterload. The concomitantly observed change in LV geometry, however, could also signify a transient ventricular-vascular stiffening with the cessation of the ductal LRS. So far, an increase of $E_{\mathrm{A}}$ and $E_{\mathrm{ES}}$ due to ventricular and vascular stiffening has been described only with age-related changes of the LV and the arterial system $(16,22,37)$.

In adaptation, LV pumping was characterized by a lower $E_{\mathrm{A}} / E_{\mathrm{ES}}$ coupling ratio secondary to high $E_{\mathrm{ES}}$. In comparision to late transition, lower $E_{\mathrm{A}}$, numerically comparable to period 1 , resulted more from higher SV than from lower BP. The $\mathrm{PDA}$ was closed in $90 \%$ of these infants. Lower $E_{\mathrm{ES}}$ compared with late transition was due to higher ESV and very likely indicated lower LV stiffness. The immature LV was remodeled.

Preterm infants with hPDA. In early transition, LV pumping was characterized by a lower $E_{\mathrm{A}} / E_{\mathrm{ES}}$ coupling ratio than in stable infants. This was secondary to low $E_{\mathrm{A}}$ and $E_{\mathrm{ES}}$ and confirms recent literature (28). $E_{\mathrm{A}}$, due to lower systolic $\mathrm{BP}$ and higher SV in hPDA (5), was significantly lower throughout transition-adaptation as was $E_{\mathrm{ES}}$, reflecting lower contractility and changed geometry of the volume-loaded LV. Accordingly, the $E_{\mathrm{A}} / E_{\mathrm{ES}}$ graph in adaptation (Figure 2) represents a marked rightward shift of the curve, as described in adult patients with heart failure $(29,30)$.

We would like to point out that, as already acknowledged in the Methods section, we might have underestimated $E_{\mathrm{ES}}$ by neglecting the increasing $V_{0}$ in late transition and adaptation. To prevent this would have required measurement of the preejection period, which has a poor interobserver repeatability in preterm infants, despite an overall good correlation in adults (38).

$E_{\mathrm{A}} / E_{\mathrm{ES}}$ graphs (Figure 2) and the corresponding $E_{\mathrm{A}}: E_{\mathrm{ES}}$ relation (Figure 3) enhance our understanding of the pathophysiology of the post ligation cardiac syndrome $(39,40)$, where the increased afterload, cessation of the LRS, and lowered LV preload lead to an acute $E_{\mathrm{A}}: E_{\mathrm{ES}}$ relation mismatch. Serial postoperative $E_{\mathrm{A}}$ and $E_{\mathrm{ES}}$ calculations, also estimated using the Teichholz methodology, revealed that $E_{\mathrm{ES}}$ improves faster than $E_{\mathrm{A}}$ after ligation of the PDA (41).

\section{Pumping and the PDA}

We consider the noticed high $E_{\mathrm{A}} / E_{\mathrm{ES}}$ coupling ratio secondary to a low $E_{\mathrm{ES}}$ in early transition and the consecutive tandem rise of both $E_{\mathrm{A}}$ and $E_{\mathrm{ES}}$ in late transition in stable preterm infants to be the most interesting result of this study. The corresponding $E_{\mathrm{A}} / E_{\mathrm{ES}}$ graphs (Figure 2 ) and the unexpectedly small variation in the SV/kg (Table 1) led us to conclude that physiological ductal patency, specifically the LRS in early and late transition, very likely enhances the augmentation of $E_{\mathrm{ES}}$ in late transition.

Actually, the LRS across the PDA causes a slight overperfusion of the entire fetal "minor" circulation extending from the pulmonary branches to the aortic isthmus, and concomitantly, it reduces systemic afterload. The PDA adds to pulmonary perfusion, and its highly oxygen-saturated blood and also vascular stretch reduce pulmonary vascular resistance. In term infants, the increased pulmonary venous return enhances closure of the preexisting second LV-filling source, the foramen ovale, and increases filling of the mature, after late gestation already adequately preloaded LV. Eventually, by the Frank-Starling mechanism, this increased LV preload increases LV contractility. The resulting augmented SV in the aorta proximal to the watershed of the isthmus is also beneficial for coronary and cerebral perfusion (42).

In preterm infants with less mature lungs, a less restrictive foramen ovale and an inadequately preloaded LV that described slight overperfusion seems to be of an even greater relevance. This training enables the immature LV to exhibit an adequate contractility $\left(E_{\mathrm{ES}}\right)$ when being subjected to the increase in $E_{\mathrm{A}}$ associated with ductal constriction.

All this, however, applies only to near-to-physiologic hemodynamics, but not to pathophysiologic hemodynamics when a large LRS across the PDA causes LV overload (Figure 2) and-by overperfusion-sometimes hemorrhagic pulmonary edema and cerebral hemorrhage (40).

Our conclusion that physiological ductal patency in transition is very likely a key factor for the evolution of LV pumping toward better contractility during transition-adaptation is based on the principles of the Frank-Starling mechanism. As explained in the introduction, the regulator of this mechanism in the sarcomere is titin $(8,13)$. Further research is needed on titin and its respective isoform myocardial expression rates in both term and preterm infants in this unique period.

There are inherent limitations of our study. This was a retrospective study without preemptive sample size calculations. Thus, nonsignificant $P$ values do not necessarily point to the absence of an effect under consideration. A point of criticism could be our methodological approach to which we have referred in detail in the Methods section. The classification by period was largely based on term infants' time frames for transition and adaptation, which may vary individually in very preterm infants. The strengths of the study include the sample size, the well-elaborated and structured setting of fEcho in our NICU with continuous computerization and simultaneously measured vital parameters, experienced examiners ensuring reliable, high-quality data, as well as the fact that by the retrospective design, examiners were unbiased when analyzing $E_{\mathrm{A}}$ and $E_{\mathrm{ES}}$.

\section{CONCLUSION}

The immature LV faces an increase of afterload after birth. We analyzed the systolic pump function of the immature LV in a cohort of very preterm infants within the transition- 
adaptation sequence by applying the $E_{\mathrm{A}} / E_{\mathrm{ES}}$ pump model in which $E_{\mathrm{A}}$ reflects the net afterload, whereas $E_{\mathrm{ES}}$ reflects the foremost contractility.

In stable preterm infants with near-to-physiologic hemodynamics, LV systolic pump function was characterized by a maximum $E_{\mathrm{A}} / E_{\mathrm{ES}}$ coupling ratio secondary to low $E_{\mathrm{ES}}$ in early transition, a tandem rise of $E_{\mathrm{A}}$ and $E_{\mathrm{ES}}$ in late transition, and a low $E_{\mathrm{A}} / E_{\mathrm{ES}}$ coupling ratio secondary to high $E_{\mathrm{ES}}$ in adaptation. These findings lead us to conclude that LV pumping evolves toward higher contractility and also that this process is enhanced by the physiological PDA.

In preterm infants with the pathophysiologic hemodynamics of a hPDA, LV systolic-pump function was characterized by significantly lower $E_{\mathrm{A}}$ and lower $E_{\mathrm{ES}}$ throughout transition-adaptation. These findings add to the understanding of the postligation syndrome when the immature overloaded LV faces an abrupt rise in systemic afterload with cessation of the PDA.

\section{SUPPLEMENTARY MATERIAL}

Supplementary material is linked to the online version of the paper at http://www.nature.com/pr

\section{ACKNOWLEDGMENTS}

We thank Dave Hill for his valuable assistance in preparation of this manuscript.

Disclosure: The authors declare no conflict of interest.

\section{REFERENCES}

1. Azhibekov T, Soleymani S, Lee BH, et al. Hemodynamic monitoring of the critically ill neonate: an eye on the future. Semin Fetal Neonatal Med 2015;20:246-54.

2. Engle WD. Blood pressure in the very low birth weight neonate. Early Hum Dev 2001;62:97-130.

3. Noori S, Seri I. Pathophysiology of newborn hypotension outside the transitional period. Early Hum Dev 2005;81:399-404.

4. El Hajjar M, Vaksmann G, Rakza T, et al. Severity of the ductal shunt: a comparison of different markers. Arch Dis Child Fetal Neonatal Ed 2005;90:F419-22.

5. Jain A, Shah PS. Diagnosis, evaluation, and management of patent ductus arteriosus in preterm neonates. JAMA Pediatr 2015;169:863-72.

6. Evans N, Kluckow M. Early ductal shunting and intraventricular haemorrhage in ventilated preterm infants. Arch Dis Child Fetal Neonatal Ed 1996;75:F183-6.

7. Finnemore A, Groves A. Physiology of the fetal and transitional circulation. Semin Fetal Neonatal Med 2015;20:210-6.

8. Hamdani N, Herwig M, Linke WA. Tampering with springs: phosphorylation of titin affecting the mechanical function of cardiomyocytes. Biophys Rev 2017;9:225-37.

9. Walker JS, de Tombe PP. Titin and the developing heart. Circ Res 2004;94:860-2.

10. Prsa M, Sun L, van Amerom J, et al. Reference ranges of blood flow in the major vessels of the normal human fetal circulation at term by phasecontrast magnetic resonance imaging. Circ Cardiovasc Imaging 2014;7: 663-70.

11. Fernandez Pineda L, Tamariz-Martel Moreno A, Maitre Azcarate MJ, et al. Contribution of Doppler atrioventricular flow waves to ventricular filling in the human fetus. Pediatr Cardiol 2000;21:422-8.

12. Bhorat I, Bagratee J, Reddy T. Gestational age-adjusted trends and reference intervals of the Modified Myocardial Performance Index (ModMPI) and its components, with its interpretation in the context of established cardiac physiological principles. Prenat Diagn 2014;34: 1031-6.

13. Tonino $\mathrm{P}$, Kiss $\mathrm{B}$, Strom J, et al. The giant protein titin regulates the length of the striated muscle thick filament. Nat Commun 2017;8:1041-9.

14. Sunagawa K, Maughan WL, Burkhoff D, et al. Left ventricular interaction with arterial load studied in isolated canine ventricle. Am J Physiol 1983;245:H773-80.

15. Starling MR. Left ventricular-arterial coupling relations in the normal human heart. Am Heart J 1993;125:1659-66.

16. Borlaug BA, Kass DA. Ventricular-vascular interaction in heart failure. Heart Fail Clin 2008;4:23-36.

17. Chantler PD, Lakatta EG. Arterial-ventricular coupling with aging and disease. Front Physiol 2012;3:90.

18. Chantler PD, Lakatta EG, Najjar SS. Arterial-ventricular coupling: mechanistic insights into cardiovascular performance at rest and during exercise. J Appl Physiol (1985) 2008;105:1342-51.

19. Tanoue $Y$, Sese A, Ueno Y, et al. Bidirectional Glenn procedure improves the mechanical efficiency of a total cavopulmonary connection in highrisk fontan candidates. Circulation 2001;103:2176-80.

20. Chowdhury SM, Butts RJ, Taylor CL, et al. Validation of noninvasive measures of left ventricular mechanics in children: a simultaneous echocardiographic and conductance catheterization study. J Am Soc Echocardiogr 2016;29:640-7.

21. Chen $\mathrm{CH}$, Fetics B, Nevo E, et al. Noninvasive single-beat determination of left ventricular end-systolic elastance in humans. J Am Coll Cardiol 2001;38:2028-34.

22. Kass DA. Age-related changes in venticular-arterial coupling: pathophysiologic implications. Heart Fail Rev 2002;7:51-62.

23. Taborsky HS. Arterial-Ventricular Coupling in Term Neonates and Infants up to 6 months, A Retrospective Data Analysis.. Vienna: Pediatric and Adolescence Medicine. Medical University of Vienna: Vienna, 2015: 69.

24. Engel J, Baumgartner S, Novak S, et al. Ventriculo-arterial coupling in children with Still's murmur. Physiol Rep 2014: 2.

25. de Boode WP, Singh Y, Gupta S, et al. Recommendations for neonatologist performed echocardiography in Europe: Consensus Statement endorsed by European Society for Paediatric Research (ESPR) and European Society for Neonatology (ESN). Pediatr Res 2016;80:465-71.

26. Teichholz LE, Kreulen T, Herman MV, et al. Problems in echocardiographic volume determinations: echocardiographic-angiographic correlations in the presence of absence of asynergy. Am J Cardiol 1976;37:7-11.

27. Kelly RP, Ting CT, Yang TM, et al. Effective arterial elastance as index of arterial vascular load in humans. Circulation 1992;86:513-21.

28. de Waal K, Phad N, Collins N, et al. Cardiac remodeling in preterm infants with prolonged exposure to a patent ductus arteriosus. Congenit Heart Dis 2017;12:364-72.

29. Ky B, French B, May Khan A, et al. Ventricular-arterial coupling, remodeling, and prognosis in chronic heart failure. J Am Coll Cardiol 2013;62:1165-72.

30. Burkhoff D. Pressure-volume loops in clinical research: a contemporary view. J Am Coll Cardiol 2013;62:1173-6.

31. Meyer S, Sander J, Graber S, et al. Agreement of invasive versus noninvasive blood pressure in preterm neonates is not dependent on birth weight or gestational age. J Paediatr Child Health 2010;46:249-54.

32. Nagasawa H, Kohno Y, Yamamoto Y, et al. Methodologic comparison of left ventricular stroke volumes in the early neonatal period by echocardiography. Pediatr Cardiol 2014;35:1415-20.

33. Burkhoff D, Sagawa K. Ventricular efficiency predicted by an analytical model. Am J Physiol 1986;250:R1021-7.

34. Khosroshahi HE, Ozkan EA, Kilic M. Arterial and left ventricular end-systolic elastance in normal children. Eur Rev Med Pharmacol Sci 2014;18:3260-6.

35. Pladys P, Wodey E, Beuchee A, et al. Left ventricle output and mean arterial blood pressure in preterm infants during the 1st day of life. Eur J Pediatr 1999;158:817-24.

36. Kluckow M, Evans N. Superior vena cava flow in newborn infants: a novel marker of systemic blood flow. Arch Dis Child Fetal Neonatal Ed 2000;82: F182-7. 
37. Antonini-Canterin F, Carerj S, Di Bello V, et al. Arterial stiffness and ventricular stiffness: a couple of diseases or a coupling disease? A review from the cardiologist's point of view. Eur J Echocardiogr 2009;10:36-43.

38. Schwarz CE, Preusche A, Baden W, et al. Repeatability of echocardiographic parameters to evaluate the hemodynamic relevance of patent ductus arteriosus in preterm infants: a prospective observational study. BMC Pediatr 2016;16:18.

39. Noori S, McNamara P, Jain A, et al. Catecholamine-resistant hypotension and myocardial performance following patent ductus arteriosus ligation. J Perinatol 2015;35:123-7.
40. El-Khuffash A, Weisz DE, McNamara PJ. Reflections of the changes in patent ductus arteriosus management during the last 10 years. Arch Dis Child Fetal Neonatal Ed 2016;101:F474-8.

41. Nagata H, Ihara K, Yamamura K, et al. Left ventricular efficiency after ligation of patent ductus arteriosus for premature infants. J Thorac Cardiovasc Surg 2013;146:1353-8.

42. Noori SSI. The very low birth weight neonate with a hemodynamically significant ductus arteriosus during the first postnatal weekIn:Polin Reds. Hemodynamics and Cardiology. Neonatology Questions and Controversies 2nd edn. New York: Columbia University Medical Center, 2008: 269-91. 